\title{
The Use of Surgical Splints in Orthognathic Surgery: A Bibliometric Study
}

\author{
Francisco Samuel Rodrigues Carvalho ${ }^{1}$ Déborah Isis de Oliveira Barbosa ${ }^{2}$ lara Furtado Torquato ${ }^{2}$ \\ André Mattos Britto de Souza ${ }^{2}$ Roberta Dalcico ${ }^{2}$ Filipe Nobre Chaves ${ }^{1}$ Fábio Wildson Gurgel Costa ${ }^{3}$ \\ 1 Postgraduate Program in Health Science and Dentistry School, \\ Federal University of Ceará (UFC) - campus Sobral, Sobral, Ceará, \\ Brazil \\ ${ }^{2}$ Dentistry Course, University of Fortaleza (UNIFOR), Fortaleza, Ceará, \\ Brazil \\ ${ }^{3}$ Department of Radiology, Graduate Program in Dentistry, Federal \\ University of Ceará (UFC), Fortaleza, Ceará, Brazil \\ Address for correspondence Filipe Nobre Chaves, DDS, PhD, Rua \\ Conselheiro José Júlio, S/N, Centro, Sobral, Ceará, CEP, 62.010-820, \\ Brazil (e-mail: filipenobrechaves@gmail.com). \\ Indian J Plast Surg 2022;55:26-30.
}

\begin{abstract}
Introduction Orthognathic surgeries require the use of surgical splints (SS) to stabilize the occlusion and the segments fixed with plates and screws. Technological advances in the field of computing and the possibility of generating three-dimensional (3D) images have brought different possibilities for making SS, which has generated greater predictability and customization of surgical plans. The bibliometric study can have a qualitative character through the scope of articles in a certain area of knowledge. It is a selection process that can track a topic or scientific production. Methods The present study aimed to carry out a bibliometric literature review, in order to assess the evolution of the use of SS and the different planning protocols in orthognathic surgery. The Scopus database was used, with the terms "splint" and "orthognathic surgery."

Keywords

- Splints

- Orthognathic Surgery

- Bibliometrics

- Dentofacial deformities

- 3D printing

- CAD/CAM

Results A total of 331 articles were found. These were exported to Rayyan for application of the inclusion and exclusion criteria and selection of articles. A total of 76 references were selected and exported to the VOSviewer application for the analysis of bibliometric data.

Conclusions Orthognathic surgery was initially not associated with any computerized technological resource; however, it underwent updates between the years 2010 to 2012. These advances allowed surgical planning to become faster, cheaper, and more accurate.
\end{abstract}

\section{Introduction}

Orthognathic surgery procedures involving the jaws require the use of surgical splints (SS) for a more reliable stabilization of the movements performed in the operated bone segments. ${ }^{1}$ An acceptable method to guide the positioning of the bone bases and their respective fixations involves the use of SS made of acrylic. ${ }^{2}$

Before internal fixation systems, it was necessary to maintain the maxillomandibular block in the postoperative period to stabilize the planned final occlusion. ${ }^{3}$ With the advent of these fixation systems, maintaining the SS in published online September 27, 2021
DOI https://doi.org/ $10.1055 / \mathrm{s}-0041-1734570$ ISSN 0970-0358.

\footnotetext{
(c) 2021. Association of Plastic Surgeons of India. All rights reserved. This is an open access article published by Thieme under the terms of the Creative Commons Attribution-NonDerivative-NonCommercial-License, permitting copying and reproduction so long as the original work is given appropriate credit. Contents may not be used for commercial purposes, or adapted, remixed, transformed or built upon. (https://creativecommons.org/ licenses/by-nc-nd/4.0/)

Thieme Medical and Scientific Publishers Pvt. Ltd., A-12, 2nd Floor, Sector 2, Noida-201301 UP, India
} 
position started to be indicated only as an adjunct tool in elastic therapy or in cases of segmental maxilla surgery. ${ }^{3-6}$

Technological advances in the field of computing and the generation of three-dimensional (3D) images have led to the creation of virtual planning protocols in orthognathic surgery. This fact allowed a change of paradigms as to how to carry out the planning, predictability, execution, and confection of the SS. ${ }^{7}$ Hence, it is a topic of paramount importance, which encourages researchers to perform future welldesigned clinical trials, assessing the accuracy of virtual planning protocols for soft-tissue outcomes in the midsagittal plane in comparison to digital profile cephalogram-based traditional surgical planning.

In analytical research, bibliometrics is defined as the use of statistical methods for the quantitative evaluation of academic literature. ${ }^{8,9}$ The benefits of this type of analysis include the evaluation of researchers or scientific publications, the study of the structure of an area of the scientific field, the identification of important topics, the discovery of new developments, among others. ${ }^{10-13}$

The bibliometric study can also have a qualitative character, seeking to cover articles in a certain area of knowledge. From this selection, we can address and generate a scientific production. As a principle, this type of research considers both relevance and methodological rigor. ${ }^{14-16}$ A database of scientific articles is selected, and from the combination of keywords and application of inclusion and exclusion criteria, an exploratory literature review is performed to create categories of analysis of the studied subject. ${ }^{14,15}$

The two most widely used bases are Scopus and Web of Science. Any comparison between them is hampered by the fact that both are in continuous development. ${ }^{16}$

There are no bibliometric studies related to the orthognathic surgery to date. Thus, the present study was performed to answer the following research question: "What are the most relevant bibliometric data of studies on the scientific field of orthognathic surgery-related surgical splints over the years?"

\section{Materials and Methods}

Study Design, Search Strategy and Selection of Articles This work is a descriptive bibliometric study, to evaluate research that addresses the topic related to SS used in orthognathic surgery. The research was performed in the Scopus database on May 9, 2020, with the search strategy TITLE-ABSKEY (splint) AND TITLE-ABS-KEY (orthognathic AND surgery). The studies found were exported to an application used to select articles in systematic reviews (Rayyan, Qatar Computing Research Institute, Doha, Qatar). ${ }^{17}$

The articles found in the search were evaluated by two examiners (DIOB and IFT) and, later, by a third reviewer (AMBS). In case of inconsistency among the three mentioned, a fourth expert reviewer on the subject (FSRC) was called in for evaluation.

Studies involving laboratory and clinical tests, observational studies, and clinical trials that evaluated SS used in orthognathic surgery were included. Studies that did not evaluate any characteristics related to the properties of the splints or did not use them were excluded.

After selecting the articles, they were exported to the VOSviewer application (version 1.6.14) for the analysis of bibliometric data (article citations, places of publication, cocitations, most cited authors, and the studies that showed the most significant results). To this end, analytical maps were created based on the bibliographic and textual data of the selected articles.

\section{Results}

We included articles published from 1975 to May 9, 2020. Three hundred and thirty-one articles were found. These were exported to Rayyan software, where inclusion and exclusion criteria were applied to titles and abstracts. At the end of the process, 76 references were obtained and analyzed in the present bibliometric review.

The five most cited articles within the sample were as follows: Swennen et al with 205 citations, Aboul-Hosn Centenero and Hernández-Alfaro with 141 citations, Metzger et al with 102 citations, Swennen et al with 90 citations, and Mavili et al with 85 citations. ${ }^{18-22}$ All articles addressed the making of SS with 3D printing, and two of them also involved virtual planning in orthognathic surgery (-Fig. 1). ${ }^{18-22}$

The selected studies were conducted in the following 25 countries: Germany $(n=16)$, United States $(n=15)$, Belgium $(n=8)$, Switzerland $(n=7)$, China $(n=7)$, Taiwan $(n=5)$, United Kingdom $(n=4)$, France $(n=3)$, Japan $(n=3)$, South Korea $(n=3)$, Spain $(n=2)$, Austria $(n=2)$, Canada $(n=2)$, Brazil $(n=2)$, Portugal $(n=1)$, Italy $(n=1)$, Thailand $(n=1)$, Hongkong $(n=1)$, West Germany $(n=1)$, Australia $(n=1)$, Denmark $(n=1)$, England $(n=1)$, and Turkey $(n=1)$. The two countries that present more work, Germany and the United States, had their main publications between the years 2010 and 2012 (-Fig. 2).

The most cited citations within the 1,139 references that are in the 76 selected articles were as follows: Gateno et al $(n=8)$, Zinser et al $(n=7)$, and Xia et al $(n=7)$. They were all related within the cluster with equivalent strength (-Fig. 3).

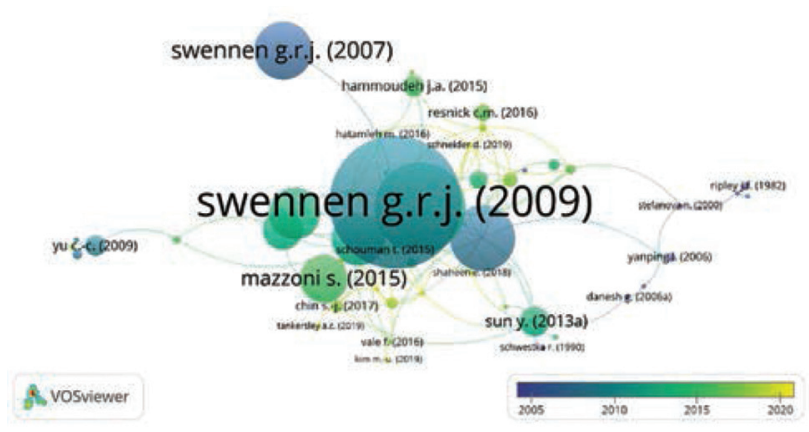

Fig. 1 Analysis of the most cited documents in the selected literature. 


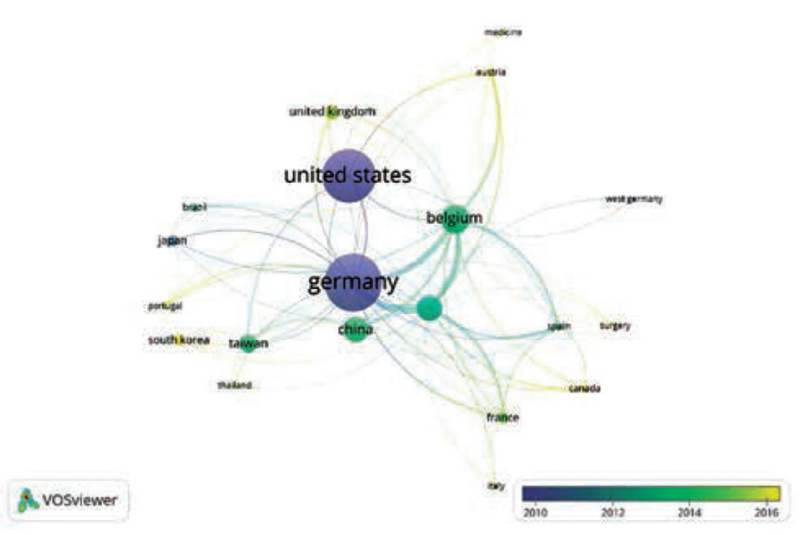

Fig. 2 Analysis of the countries most cited in the selected literature.

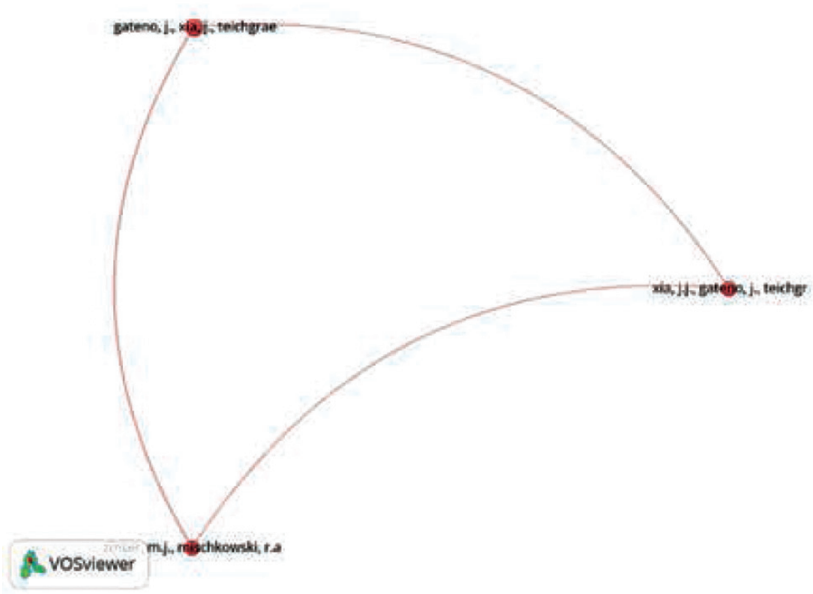

Fig. 3 Analysis of the most referenced co-citations in the selected literature.

The most referenced authors were as follows: Gateno et al $(n=125)$, Teichgraeber et al $(n=97)$, Xia et al $(n=89)$, Schutyser et al $(n=43)$, and Zinser et al $(n=43)$. All of these were related within the cluster, however Gateno et al, Teichgraeber et al, and Xia et al, presented the greatest strengths of the connections (-Fig. 4).

The analysis of databased on titles and abstracts led to the identification of the most common terms. Of the 1,724 terms, $22 \mathrm{had}$ a minimum number of 10 occurrences. For each of the 22 terms, a relevance score was calculated. Based on this score, the most relevant terms were selected. The standard for choosing these terms was $60 \%$ relevant. Therefore, 13 terms were selected: orthognathic surgery $(n=55)$, splint $(n=49)$, planning $(n=45)$, patient $(n=41)$, study $(n=36)$, computer $(n=29)$, model $(n=23)$, surgical splint $(n=21)$, model of surgery $(n=17)$, CAD/CAM $(n=16)$, time $(n=16)$, case $(n=16)$, and surgeon $(n=11)$, distributed in three clusters (-Fig. 5). The greatest relevance was observed in orthognathic surgery (1.84), splint (1.27), and planning (1.36).

-Fig. 5 illustrates a network visualization of the terms used in the present search strategy. Circles represent keywords, and their colors represent specific years (blue $=2011$; green $=2013$; yellow $=2015$ ); lines represent the distance between words, and the closer two words are located to each other, the

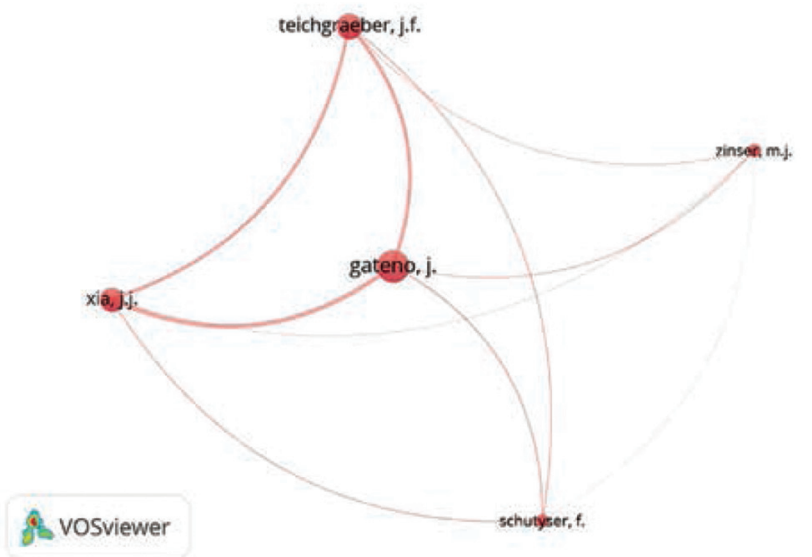

Fig. 4 Analysis of the most cited authors in the selected literature.

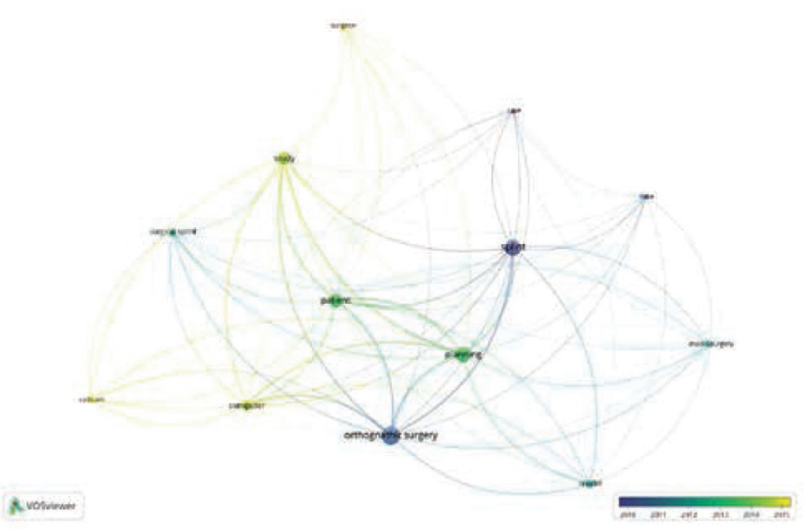

Fig. 5 Analysis of the most cited words in the selected literature.

stronger their relatedness. In 2011, the circles with higher weights were related to the terms "splint," "orthognathic surgery," "model surgery," and "time." The year 2013 is represented by the color green, in which surgical planning is geared to the patient and not model surgery. Meanwhile, yellow, the color of which represents 2015, was the time when studies focused on computer and computer-aided design (CAD)/computer-aided manufacturing (CAM) technology. Thus, demonstrating a chronological order of research aimed at orthognathic surgery and its technological evolution.

\section{Discussion}

The articles most cited in the studies are by Gateno et al, Xia et al, and Zinser et al. ${ }^{23-25}$ These authors are the precursors of virtual planning and SS obtained from 3D technologies for orthognathic surgery. ${ }^{23,25}$ For this reason, citation rates are high among these and many other articles. When studying orthognathic surgery, these research works are the most commonly used as references. These studies justify why, in contemporary times, plaster model surgery and the manufacture of conventional SS are being less used. Despite being a relatively new advent, it is already common in this type of procedure, due to its precision and reproducibility. $7,19,26-28$ 
The study by Gateno et $\mathrm{al}^{23}$ demonstrated a positive result of stereolithographic splints, generated using CAD/CAM, thereby obtaining a high degree of precision. No differences were observed between SS made conventionally and those made in a 3D system. Both the conventional method and those obtained from the stereolithography (STL) file showed the same precision. The adjustments made to the splints obtained from STL files were the same as for the conventionally made splints. ${ }^{23,29-33}$

Thus, it is concluded that both ways of obtaining different SS are viable for use in orthognathic surgery. With the recent development of the 3D system in surgical planning technology, it is possible to carry out the entire planning of the surgery and the preparation of the splints in a computational environment, thus facilitating the process for the maxillofacial surgeon. Also, there is the possibility of better predictability of the final result. ${ }^{23,29-33}$

Many unwanted outcomes are the result of poor planning. Thus, with the need to improve the surgical planning of craniomaxillofacial deformities, a 3D computer-assisted surgical simulation system (CASS) was developed. In this system, it is possible to perform the surgery virtually and create a prediction of the outcome in the patient. In addition to twodimensional (2D) and 3D measurements, the software is also capable of performing volumetric measurements. ${ }^{32}$

The results of the study by Xia et al showed that the CASS method had a lower cost in terms of the surgeon's time, patient's time, and material costs. ${ }^{24,34-36}$ Also, he reported that the program encounters difficulty in establishing the maximum intercuspation (MI) function on the computer. It is almost impossible to be sure that what is being observed on the computer screen is, in fact, the representation of the best real alignment. To ensure that the final digital occlusion is in correct MI, plaster models that are physically positioned in the final occlusion must be digitized.

Xia et al pointed out several problems associated with the traditional planning system, thus justifying that the best option for orthognathic surgeries in virtual planning. In the conventional method, the analysis of facial photographs had to be done two-dimensionally. However, by virtue of advances in computing, it is now possible to perform these analyzes in a 3D way. ${ }^{24}$

Despite this, some measures are generally difficult to interpret, since those that are in 3D are translated directly (expanded) into the original 2D form. Another problem with 3D cephalometry is the uncertainty of how angles are constructed by planes. ${ }^{24}$

The complete production of surgical splints on 3D printers is often less time consuming. Personalized splints reduce the duration of surgery, anesthesia time, and costs. Thus, direct communication with prosthetist is possible, allowing quick changes if necessary. ${ }^{36}$

There are technical limitations regarding the use of 3D printing. There is a learning curve for developing proficiency, using software planning, which is offset by increased surgical predictability and reduced time, making this a worthwhile investment for planning surgeries. ${ }^{36}$

The 3D printing technique presents a higher dose of radiation due to radiographic examinations. However, if the number of tests is reduced, there will also be a decrease in exposure as well as in the time spent. This is possible due to the cone-beam CT technique, which has a reduced dose of radiation and better quality images. ${ }^{34}$

A great advantage of virtual methods is the reduction of time spent on surgical planning. Despite being a higher value process, compared with the conventional technique, the investment in software is of great value, as the number of patients undergoing treatment increases and the cost is compensated. Besides, the 3D technique has better predictability. ${ }^{34}$

On the other hand, for general planning, the 3D technique is the one that demands the most time, as the steps are timeconsuming, since they involve a more advanced cephalometric system, heavier data files, and a segmentation process with an added dimension. ${ }^{34}$

However, in the study by Resnick et al, virtual surgical planning was cheaper and less time consuming than in the standard form for all types of cases analyzed. ${ }^{35}$ The greatest differences in time and cost were seen concerning the asymmetry, as it is difficult to quantify this inequality in $2 \mathrm{D}$ and the increased complexity of the surgical model for these cases, compared with the ease of viewing and altering 3D images in virtual planning.

By eliminating facial arch transfer, there is a significant reduction in the surgeon's time compared with the traditional planning method. After the patient's CT is completed, the preparation of the computer data, including the digitalization of the dental model, segmentation of CT images, 3D model reconstruction, skull model creation, digitization of the markings, and virtual osteotomies, are completed by a centralized service. ${ }^{24}$

Zinser et al developed and patented (WO 2008/031562) CAD/CAM for the manufacture of multiple surgical splints for orthognathic surgery. ${ }^{25}$ The benefits of this technique are that it allows the direct operating room sharing of virtual surgical plans, in addition to being easy to use, relatively inexpensive, and clinically efficient.

The use of CAD/CAM technology provided a precise and clinically acceptable transfer from preoperative planning to the surgical environment, comparing favorably with the variations reported in conventional clinical protocols that incorporate cephalometric analysis, face transfer to semi-adjustable articulator, and model surgery plasterboard. $^{25}$

Virtual planning, using CAD/CAM technology or CASS methods, requires the use of specific equipment that has a cost associated with its acquisition. However, considering that it is a durable equipment, this has its cost reduced throughout the time of use. ${ }^{25,35}$ The authors of the present study suppose that CASS may be better because of less time spent by the various professionals which may actually be more cost-effective.

\section{Conclusion}

Advances in orthognathic surgery have allowed surgical planning to be more patient-oriented and more individualized. Besides, contemporary studies are more related to 
technology using computers as well as the advent of SS manufactured using the CAD/CAM system, which is currently the best option of choice for surgeons, due to its high level of accuracy, its low cost, its better predictability of results, and its fast processing.

\section{Conflict of Interest and Funding None.}

\section{References}

1 Ripley JF, Steed DL, Flanary CM. A composite surgical splint for dual arch orthognathic surgery. J Oral Maxillofac Surg 1982;40 (10):687-688

2 Bell CNA. Prevention of tooth decalcification under acrylic splints used in orthognathic surgery. J Oral Maxillofac Surg 1985;43(08): 650-651

3 Block MS, Hoffman D. A removable orthognathic surgical splint. J Oral Maxillofac Surg 1987;45(02):195

4 Chow TK, Bok WS. New surgical splint for segmental maxillary osteotomies. J Oral Maxillofac Surg 1993;51(01):97-98

5 Ismail M, Wessel J, Farrell B. Maintenance of segmental maxillary expansion: the use of custom, virtually designed, and manufactured palatal appliances without the use of an occlusal splint. J Oral Maxillofac Surg 2019;77(07):1468.e1-1468.e8

6 Stokbro K, Aagaard E, Torkov P, Marcussen L, Bell RB, Thygesen T. Surgical splint design influences transverse expansion in segmental maxillary osteotomies. J Oral Maxillofac Surg 2017;75 (06):1249-1256

7 Zinser MJ, Sailer HF, Ritter L, Braumann B, Maegele M, Züller JE. A paradigm shift in orthognathic surgery? A comparison of navigation, computer-aided designed/computer-aided manufactured splints, and "classic" intermaxillary splints to surgical transfer of virtual orthognathic planning. J Oral Maxillofac Surg 2013;71 (12):2151.e1-2151.e21

8 Cobo MJ, López-Herrera AG, Herrera-Viedma E, Herrera F. An approach for detecting, quantifying, and visualizing the evolution of a research field: a practical application to the Fuzzy Sets Theory field. J Informetrics 2011;5(01):146-166

9 Cobo MJ, Martínez MA, Gutiérrez-Salcedo M, et al. 25years at Knowledge-Based Systems: a bibliometric analysis. Knowl Base Syst 2015;80:3-13

10 Chen XL, Chen BY, Zhang CX, Hao TY. Discovering the recent research in natural language processing field based on a statistical approach. Lect Notes Comput Sci 2017;10676:507-517

11 Wallace ML, Larivière V, Gingras Y. A small world of citations? The influence of collaboration networks on citation practices. PLoS One 2012;7(03):e33339

12 Chen X, Weng M, Hao A. Data-driven approach for discovering the recent research status of diabetes in China. Lect Notes Comput Sci 2017; 10594:89-101

13 Diem A, Wolter SC. The use of bibliometrics to measure research performance in education sciences. Res High Educ 2013;54(01): 86-114

14 Okubo Y. Bibliometric Indicators and Analysis of Research Systems.: Methods and Examples, OECD Science, Technology and Industry Working Papers, No. 1997/01. OECD Publishing 1997

15 Torraco R. Writing integrative literature reviews: guidelines and examples. Hum Resour Dev Rev 2005;4:356-367

16 Moed HF. New developments in the use of citation analysis in research evaluation. Arch Immunol Ther Exp (Warsz 2009;57(01): $13-18$

17 Ouzzani M, Hammady H, Fedorowicz Z, Elmagarmid A. Rayyan-a web and mobile app for systematic reviews. Syst Rev 2016;5(01): 210
18 Swennen GR, Mollemans W, Schutyser F. Three-dimensional treatment planning of orthognathic surgery in the era of virtual imaging. J Oral Maxillofac Surg 2009;67(10):2080-2092

19 Aboul-Hosn Centenero S, Hernández-Alfaro F. 3D planning in orthognathic surgery: CAD/CAM surgical splints and prediction of the soft and hard tissues results - our experience in 16 cases. J Craniomaxillofac Surg 2012;40(02):162-168

20 Metzger MC, Hohlweg-Majert B, Schwarz U, Teschner M, Hammer B, Schmelzeisen R. Manufacturing splints for orthognathic surgery using a three-dimensional printer. Oral Surg Oral Med Oral Pathol Oral Radiol Endod 2008;105(02):e1-e7

21 Swennen GR, Barth EL, Eulzer C, Schutyser F. The use of a new 3D splint and double CT scan procedure to obtain an accurate anatomic virtual augmented model of the skull. Int J Oral Maxillofac Surg 2007;36(02):146-152

22 Mavili ME, Canter HI, Saglam-Aydinatay B, Kamaci S, Kocadereli I. Use of three-dimensional medical modeling methods for precise planning of orthognathic surgery. J Craniofac Surg 2007;18(04): 740-747

23 Gateno J, Xia J, Teichgraeber JF, Rosen A, Hultgren B, Vadnais T. The precision of computer-generated surgical splints. J Oral Maxillofac Surg 2003;61(07):814-817

24 Xia JJ, Gateno J, Teichgraeber JF. New clinical protocol to evaluate craniomaxillofacial deformity and plan surgical correction. J Oral Maxillofac Surg 2009;67(10):2093-2106

25 Zinser MJ, Mischkowski RA, Sailer HF, Züller JE. Computerassisted orthognathic surgery: feasibility study using multiple CAD/CAM surgical splints. Oral Surg Oral Med Oral Pathol Oral Radiol 2012;113(05):673-687

26 Chapuis J, Ryan P, Blaeuer M, et al. A new approach for 3D computer-assisted orthognathic surgery-first clinical case. In: Lemke HU, Inamura K, Doi K, Vannier MW, Farman AG, eds. International Congress Series. 2005:1217-1222

27 Kim MU, Kim YH, Kim YS. A study of orthognathic surgical splints development using 3D convergence technology. J Korean Soc Precis Eng 2019;36(03):301-309

28 Zinser M,Zoeller J. Computer-designed splints for surgical transfer of 3D orthognathic planning. Facial Plast Surg 2015;31(05):474-490

29 Aboul-Hosn Centenero S. 3D planning and use of computer aided design/computed aided manufacturing surgical splints in orthognathic surgery. Rev Esp Cir Oral Maxilac 2014;36(03):108-112

30 Adolphs N, Liu W, Keeve E, Hoffmeister B. RapidSplint: virtual splint generation for orthognathic surgery - results of a pilot series. Comput Aided Surg 2014;19(1-3):20-28

31 Hsu SS, GatenoJ, Bell RB, et al. Accuracy of a computer-aided surgical simulation protocol for orthognathic surgery: a prospective multicenter study. J Oral Maxillofac Surg 2013;71(01):128-142

32 Yuan P, Ho DCY, Chang CM, et al. A novel computer-aided surgical simulation (CASS) system to streamline orthognathic surgical planning. In: Liao H, Zheng G, Lee SL, Cattin P, Jannin P. Medical Imaging and Augmented Reality - 7th International Conference, MIAR 2016, Proceedings Springer-Verlag; 2016:3-14

33 Yuan P, Mai H, Li J, et al. Design, development and clinical validation of computer-aided surgical simulation system for streamlined orthognathic surgical planning. Int J CARS 2017;12 (12):2129-2143

34 Bengtsson M, Wall G, Becktor JP, Rasmusson L. A comparison of cost-effectiveness of computer-assisted 2-and 3-dimensional planning techniques in orthognathic surgery. $\mathrm{Br} \mathrm{J}$ Oral Maxillofac Surg 2019;57(04):352-358

35 Resnick CM, Inverso G, Wrzosek M, Padwa BL, Kaban LB, Peacock $\mathrm{ZS}$. Is there a difference in cost between standard and virtual surgical planning for orthognathic surgery? J Oral Maxillofac Surg 2016;74(09):1827-1833

36 McAllister P, Watson M, Burke E. A cost-effective, in-house, positioning and cutting guide system for orthognathic surgery. J Maxillofac Oral Surg 2018;17(01):112-114 